\title{
Short-period mesospheric gravity waves and their sources at the South Pole
}

\author{
Dhvanit Mehta $^{1}$, Andrew J. Gerrard ${ }^{1}$, Yusuke Ebihara ${ }^{2}$, Allan T. Weatherwax ${ }^{3}$, and Louis J. Lanzerotti ${ }^{1}$ \\ ${ }^{1}$ Center for Solar-Terrestrial Research, New Jersey Institute of Technology, 323 Martin Luther King Jr. Boulevard, \\ 101 Tiernan Hall, Newark, NJ 07102-1982, USA \\ ${ }^{2}$ Research Institute for Sustainable Humanosphere, Kyoto University, Gokasho, Uji City, Kyoto Prefecture, Japan \\ ${ }^{3}$ Merrimack College, 315 Turnpike St, North Andover, MA 01845, USA \\ Correspondence to: Dhvanit Mehta (dm36@njit.edu)
}

Received: 22 March 2016 - Published in Atmos. Chem. Phys. Discuss.: 10 May 2016

Revised: 14 September 2016 - Accepted: 10 October 2016 - Published: 20 January 2017

\begin{abstract}
The sourcing locations and mechanisms for shortperiod, upward-propagating gravity waves at high polar latitudes remain largely unknown. Using all-sky imager data from the Amundsen-Scott South Pole Station, we determine the spatial and temporal characteristics of 94 observed smallscale waves in 3 austral winter months in 2003 and 2004. These data, together with background atmospheres from synoptic and/or climatological empirical models, are used to model gravity wave propagation from the polar mesosphere to each wave's source using a ray-tracing model. Our results provide a compelling case that a significant proportion of the observed waves are launched in several discrete layers in the tropopause and/or stratosphere. Analyses of synoptic geopotentials and temperatures indicate that wave formation is a result of baroclinic instability processes in the stratosphere and the interaction of planetary waves with the background wind fields in the tropopause. These results are significant for defining the influences of the polar vortex on the production of these small-scale, upward-propagating gravity waves at the highest polar latitudes.
\end{abstract}

\section{Introduction}

The breaking and induced drag caused by atmospheric gravity waves plays an important role in the dynamics of the mesosphere-lower thermosphere (MLT) region (Fritts and Alexander, 2003). The impacts of such wave breaking are felt on a climatological scale; e.g., gravity waves fundamentally drive a meridional circulation resulting in a cool summer mesopause and warm winter mesopause (Meriwether and Gerrard, 2004). On the synoptic scale the effects of gravity waves can be seen in the localized destruction of mesospheric clouds (Gerrard et al., 2002, 2004), mesospheric fronts/bores (Brown et al., 2004), and localized wave ducting (Li et al., 2012). As such, because of their significance to the dynamics of the middle atmosphere, gravity waves have been a focus of active and ongoing research, particularly at high latitudes. However, while some studies have looked at gravity waves near the poles (Chu et al., 2011; Chen et al., 2013; Kaifler et al., 2015), observations at high latitudes are often difficult to obtain due to experimental logistics. This is even more of an issue in the Antarctic region, where few manned stations exist to operate gravity wave instrumentation during the austral winter.

Of particular interest to this study is the determination of high-latitude gravity wave source regions. Many studies have investigated the excitation of gravity waves in the lower atmosphere (Sato and Yoshiki, 2008; Gerrard et al., 2011; Moffat-Griffin et al., 2011), directly in the MLT region from auroral heating (Oyama and Watkins, 2012), and on the characteristics and seasonal variation of gravity waves in the polar MLT region (Nielsen et al., 2012; Suzuki et al., 2011). While the excitation and propagation of gravity waves during disturbed conditions, such as during sudden stratospheric warmings and stratospheric temperature enhancements (Meriwether and Gerrard, 2004), have been investigated by Wang and Alexander (2009), Yamashita et al. (2010), and Gerrard et al. (2011), there is a significant gap 
in understanding of wave generation during quiet conditions or from a climatological or quasi-climatological perspective.

One dominant gravity wave source region known to occur at polar latitudes is the polar vortex (Duck et al., 1998; Whiteway and Duck, 1999). Displacement of the polar vortex away from its mean position over a pole can result in a vertically slanted, tilted wind structure that can give rise to baroclinic instabilities (Tanaka and Tokinaga, 2002). These instabilities have been studied as a generating mechanism for larger-scale (on the order of several hundred kilometers) gravity waves through extensive modeling (Fairlie et al., 1990; O'Sullivan and Dunkerton, 1995; Plougonven and Snyder, 2007; Lin and Zhang, 2008) and observational (Guest et al., 2000; Plougonven et al., 2003; Lane et al., 2004; Gerrard et al., 2011) efforts, but to date their status as a source of small-scale gravity waves $(<100 \mathrm{~km})$ has not been investigated.

In this paper we show gravity wave observations from South Pole Station, Antarctica (hereafter SPA), from a dataset previously presented in Suzuki et al. (2011). We then model the propagation of the observed waves from their site of observation above SPA to their lower-altitude sources using ray-tracing techniques. We then analyze the potential source regions of the waves using lower atmospheric analyses. In Sect. 2 we present our gravity wave observations. In Sect. 3, the results of our ray-tracing model runs are presented, with results showing stratified layers of gravity wave sources in a region around the SPA site tightly restricted in latitude. In Sect. 4, we show lower atmospheric analyses that support the results of our modeling efforts and our interpretation of baroclinic instability as the primary mechanism of gravity wave generation by the polar vortex. Finally, we present conclusions in Sect. 5, with a discussion as to the challenges and limitations of our investigation.

\section{Gravity wave observations}

For this study we utilized data obtained from a multiwavelength all-sky imager located at SPA, originally constructed and operated by the National Institute of Polar Research (NIPR), and now operated by the Research Institute for Sustainable Humanosphere (RISH) of Kyoto University, Japan, in collaboration with the New Jersey Institute of Technology (NJIT) (Ejiri et al., 1999; Suzuki et al., 2011). The imager consists of a fish-eye lens providing a $180^{\circ}$ field of view (Nikkor $f=6 \mathrm{~mm}, \mathrm{~F} 1.4$ ), a rotating filter wheel with five filters $(427.8,557.7,630.0,589.0,486.1 \mathrm{~nm})$ for both auroral and airglow observations, and a temperature-controlled charge-coupled device (CCD) camera with $512 \times 512$ pixel resolution. Due to its location at SPA, the system is able to operate more or less continuously during the austral winter period, between April and August barring periods where the moon is at a high-elevation angle. In this paper we chiefly focused on the green line OI $(557.7 \mathrm{~nm})$ and $\mathrm{Na}(589.0 \mathrm{~nm})$ airglow filters. For data shown from 2003 and 2004, Na images have $64 \mathrm{~s}$ exposure times and are taken roughly $100 \mathrm{~s}$ apart, while green line images are taken with $8 \mathrm{~s}$ exposures, also at $100 \mathrm{~s}$ sampling rate.

Gravity wave observations have previously been reported with this instrument using its $\mathrm{Na}$ airglow filter for the 20032005 austral winters by Suzuki et al. (2011), providing a climatology of waves observed at $\sim 95 \mathrm{~km}$ for both larger-scale "band" events as well as smaller-scale "ripple" events that are commonly thought to be localized convective or dynamical instability processes. For our own analysis, we used a portion of this dataset covering July 2003, August 2003, and August 2004 as these periods showed the highest continuous $\mathrm{Na}$ airglow observations with minimal contamination by auroral emissions. Note that while the $589.3 \mathrm{~nm}$ emission is generally not sensitive to auroral contamination, we nonetheless found the presence of auroral emissions in our image data, likely as a result of spectral leakage due to complications with the filter. While this contamination was only problematic during periods where the auroral emissions were particularly bright, its persistence throughout the data set meant we were forced to compare our images with roughly simultaneous green-line $557.7 \mathrm{~nm}$ filter images taken from the same instrument. This allowed us a greater accuracy in differentiating between auroral processes and gravity wave signatures in our $\mathrm{Na}$ images and allowed us to observe gravity waves even in conditions where portions of the image were contaminated.

Prior to analyzing images for the signatures of gravity waves, it was necessary to apply a number of post-processing techniques to the data. First, to correct for distortion of the image as a result of the fish-eye lens, images were unwarped using the technique described in Garcia et al. (1997) into geographic coordinates from the original "warped" image coordinate frame. Next, the resultant images were timedifferenced in order to heighten image contrast and make it possible to identify gravity wave structure in the fairly faint airglow emission. Finally, the images were band pass filtered. While many studies using newer imager systems eschew time-differencing due to the potential introduction of artifacts, it was necessary in our analysis due to the faintness of the emission, as well as the significant difference in contrast between airglow and auroral contamination any time contamination was present. Once the images were fully processed, images were inspected for the presence of gravity waves and their observed horizontal wavelengths, periods, and propagation directions were measured and recorded.

From the 38 days of available data during July 2003, August 2003, and August 2004, we observed 94 total wave events. Examples are shown in Figs. 1 and 2. In Fig. 1, for 6 August 2004, a gravity wave is seen propagating southward at $207 \pm 6^{\circ}$ with $\lambda_{h}=17 \pm 1 \mathrm{~km}$ and $T_{\mathrm{obs}}=8 \pm 1 \mathrm{~min}$ beginning around 11:37 UT and leaving the imager field of view (FOV) at 12:07 UT (where "North" here is defined as being along $0^{\circ}$ longitude by convention). Figure 2 , for $18 \mathrm{Au}-$ 


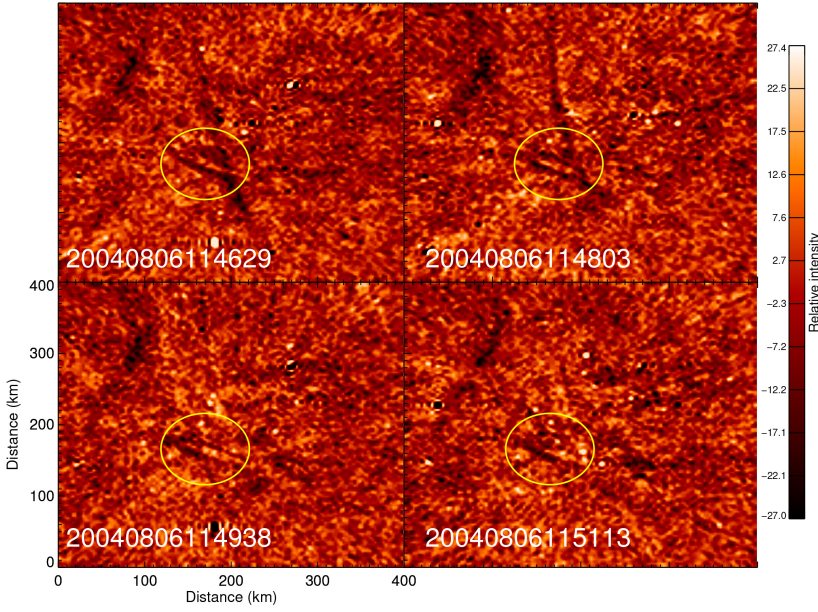

Figure 1. Processed Na image from 6 August 2004. The images were unwarped onto a $400 \times 400 \mathrm{~km}$ geographic grid (shown in the bottom left image) with the positive $y$ axis corresponding to $0^{\circ}$ longitude. Yellow circles mark the location of the observed wave in each image. Time stamps are shown in the bottom left of each image, and are read as YYYYMMDDHHMMSS. The sequence of images starts at the top left, and follows to the top right, bottom left, and finally bottom right.

gust 2004, shows a gravity wave propagating southward at $157 \pm 6^{\circ}$ with $\lambda_{h}=16 \pm 1 \mathrm{~km}$ and $T_{\mathrm{obs}}=8 \pm 1 \mathrm{~min}$, first appearing at 21:54 UT and departing from the imager FOV at 22:32 UT.

We then proceeded to perform an initial series of raytracing runs using these two waves. Our goal was 2-fold: first as a proof of concept for the application of the ray-tracing model to waves in the polar MLT, and second to demonstrate the need to run the model on an atmospheric background with synoptic-scale variation. Following this, we performed raytracing model runs on the remainder of the gravity waves in the dataset.

\section{Gravity wave source determination using the GROGRAT ray-tracing model}

Ray-tracing techniques have been applied for decades in modeling the propagation of waves through the atmosphere (Lighthill, 1978). Dunkerton and Butchart (1984) used a simple hydrostatic ray-tracing scheme to show that meridional asymmetry in the background flow due to a sudden stratospheric warming led to regions through which stationary gravity waves with horizontal wavelengths between 50 and $200 \mathrm{~km}$ could not propagate due to critical level filtering. The development of a full, three-dimensional nonhydrostatic (i.e., one in which $\frac{\partial p^{\prime}}{\partial z}+\rho g \neq 0$ ) ray-tracing algorithm by Marks and Eckermann (1995), and their subsequent additions in Eckermann and Marks (1997), led to the Gravity Wave Regional or Global Tracer (GROGRAT) ray-tracing model. The

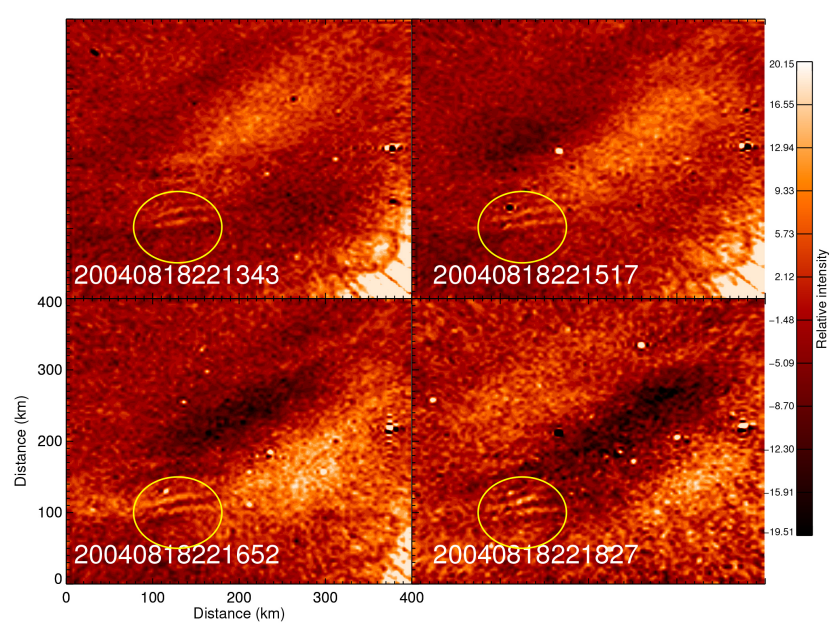

Figure 2. Processed Na image from 18 August 2004. The images were unwarped onto a $400 \times 400 \mathrm{~km}$ geographic grid (shown in the bottom left image) with the positive $y$ axis corresponding to $0^{\circ}$ longitude. Yellow circles mark the location of the observed wave in each image. Time stamps are shown in the bottom left of each image, and are read as YYYYMMDDHHMMSS. The sequence of images starts at the top left, and follows to the top right, bottom left, and finally bottom right.

model tracks the amplitude evolution and four-dimensional propagation of a wave through a background atmosphere and includes terms for radiative dissipation, amplitude saturation, and turbulent diffusion, with an upper altitude limit of $120 \mathrm{~km}$. The model utilizes an internal regridding scheme that permits the use of practically any input background atmosphere, allowing for the incorporation of multiple atmospheric data products into a single run regardless of their original grid.

GROGRAT has been used in a number of studies of wave propagation, both running in reverse for the purpose of determining tropospheric wave sources (Gerrard et al., 2004; Brown et al., 2004; Vadas et al., 2009) and for forward modeling (Lin and Zhang, 2008; Yamashita et al., 2013) the ray propagation from baroclinic regions or during disturbed conditions, such as during sudden stratospheric warmings. Raytracing analysis has previously been applied to the highlatitude MLT by Yamashita et al. (2013) in their study of gravity wave propagation during sudden stratospheric warming events, albeit with an arbitrary spectrum of waves originating in the troposphere and propagating into the middle atmosphere under varying background conditions. For our analysis of wave sources over SPA, we also utilized GROGRAT v2.9, with a grid displaced $4^{\circ}$ latitude from SPA. This avoids complications around the pole arising from the singularity at $-90^{\circ}$ latitude. We ran the model on a global $2.5^{\circ} \times 2.5^{\circ}$ spatial grid with 50 altitude levels spaced $2 \mathrm{~km}$ apart centered over the SPA site.

An important consideration in applying reverse ray-tracing techniques to gravity wave propagation through the atmo- 

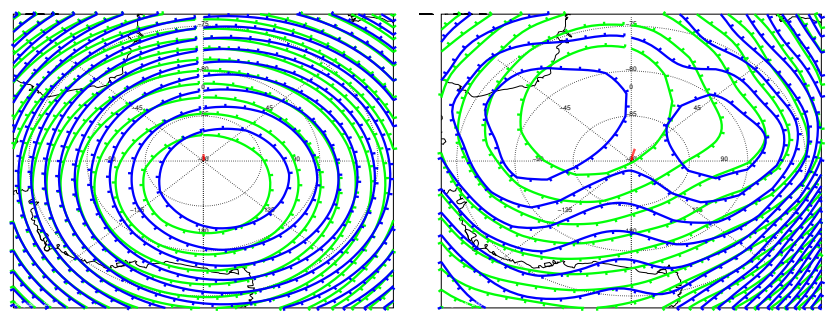

Figure 3. (Left) Results of the GROGRAT "climatological" run for the wave observed on 6 August 2004 using background pressures, temperatures, and horizontal winds reconstructed from NRLMSISE-00 and HWM-93. (Right) Results of the GROGRAT run for the same wave using an atmosphere constructed from ECMWF Reanalysis below $50 \mathrm{~km}$ altitude and NRLMSISE-00 and HWM-93 between 50 and $100 \mathrm{~km}$ altitude. The two contours in each panel represent geopotential heights at 3 mbar (blue) and $10 \mathrm{mbar}$ (green), and the red line in each panel represents the wave ray path.

sphere is the construction of an accurate atmospheric background through which the wave ray path is integrated. Two options were investigated and are presented in example runs for the waves shown in Figs. 1 and 2. The first is a purely "climatological" atmosphere and the second is an atmosphere that incorporates synoptic variation below $50 \mathrm{~km}$. "Climatological" runs used a background atmosphere constructed from the Navy Research Laboratory Mass Spectrometer and Incoherent Scatter Radar (NRLMSISE-00) (Picone et al., 2002) empirical atmospheric model and the Horizontal Wind Model (HWM-93), an empirical horizontal neutral wind model of the upper atmosphere (Hedin et al., 1996), for the entire atmosphere from the surface to $120 \mathrm{~km}$ altitude. "Synoptic" runs utilized the European Centre for Medium-Range Weather Forecasts (ECMWF) Tropical Ocean and Global Atmosphere (TOGA) (European Centre for Medium-Range Weather Forecasts, 1990) 2.5 Global Surface and Upper Air Analysis datasets below $50 \mathrm{~km}$, with NRLMSISE-00 and HWM-93 input from 50 to $100 \mathrm{~km}$, where the background atmospheric parameters were smoothed using a cubic spline fit to prevent artificial wind shears and similar features at the boundary at $50 \mathrm{~km}$. Gravity waves were initiated at $95 \mathrm{~km}$ with prescribed spatial and temporal characteristics as determined by our analysis of the all-sky imager data. The results for the wave observed on 6 August 2004 are shown in Fig. 3a and $\mathrm{b}$ for the climatological and synoptic runs, respectively. Those for 18 August 2004 are shown in Fig. 4a and b for the climatological and synoptic runs, respectively.

For the 6 August wave, both types of runs show gravity wave rays terminating in the troposphere, at $7 \mathrm{~km}$ altitude for the climatological run and at the surface for the synoptic run. However, the ray paths for the two model runs differ significantly in both direction of propagation and distance from SPA. During this period, the polar vortex, through which the wave propagates, is fairly stable, as seen in the NRLMSISE00 background in Fig. 3a, while the shape of the vortex seen
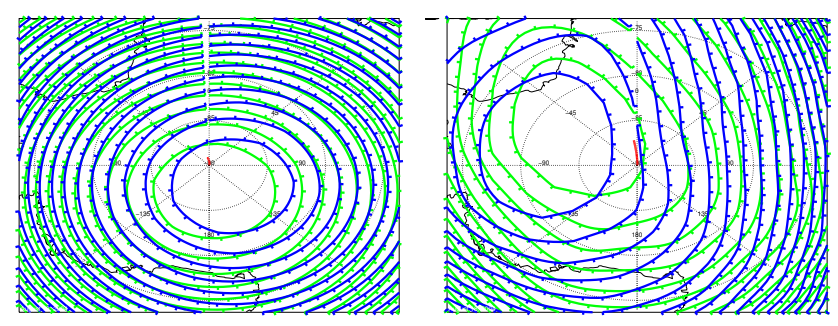

Figure 4. (Left) Results of the GROGRAT "climatological" run for the wave observed on 18 August 2004 using background pressures, temperatures, and horizontal winds reconstructed from NRLMSISE-00 and HWM-93. (Right) Results of the GROGRAT run for the same wave using an atmosphere constructed from ECMWF Reanalysis below $50 \mathrm{~km}$ altitude and NRLMSISE-00 and HWM-93 between 50 and $100 \mathrm{~km}$ altitude. The two contours in each panel represent geopotential heights at $3 \mathrm{mbar}$ (blue) and $10 \mathrm{mbar}$ (green), and the red line in each panel represents the wave ray path.

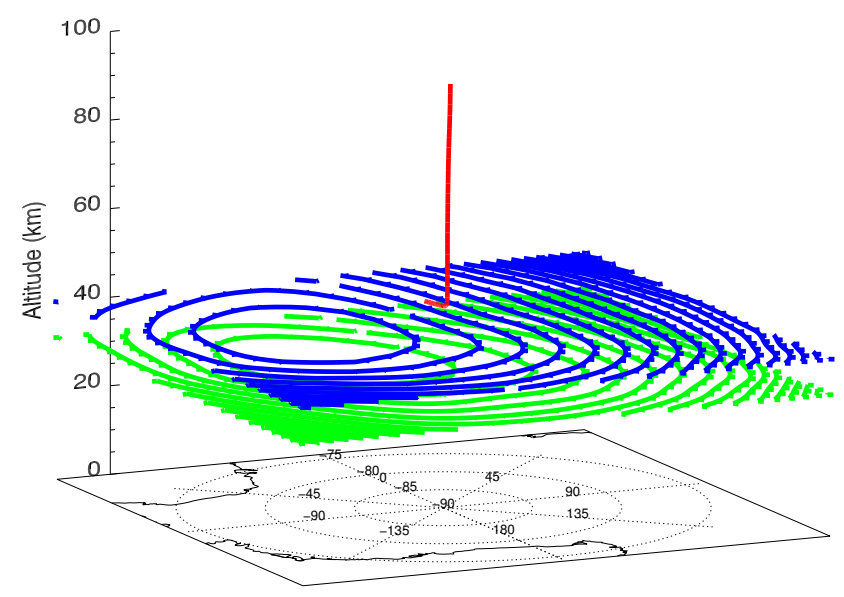

Figure 5. GROGRAT ray-tracing results for the 19 August 2004 wave shown in Fig. 4 (right) projected in three dimensions over Antarctica. The two contours represent geopotential heights at 3 mbar (blue) and 10 mbar (green), and show the wave ray path (red line).

in the ECMWF background in Fig. 3b is distorted by apparent interaction with a planetary wave.

A different result is seen for the wave observed on $18 \mathrm{Au}$ gust. The climatological run once again produces a ray path stopping in the troposphere near SPA at an altitude of $7 \mathrm{~km}$. In the ECMWF-based synoptic model run the ray path travels down into the stratosphere, where it travels farther out than for the climatological run, before stopping at a height of $42.5 \mathrm{~km}$ at roughly $3.5^{\circ}$ latitude from SPA. The polar vortex is displaced away from its normal configuration centered close to SPA and tilted in the region where the wave is determined to originate. This can be seen more clearly in the three-dimensional projection shown in Fig. 5, which is a projection of the two-dimensional plot shown in Fig. 4 (right). Typical uncertainties in the model results arising from uncer- 

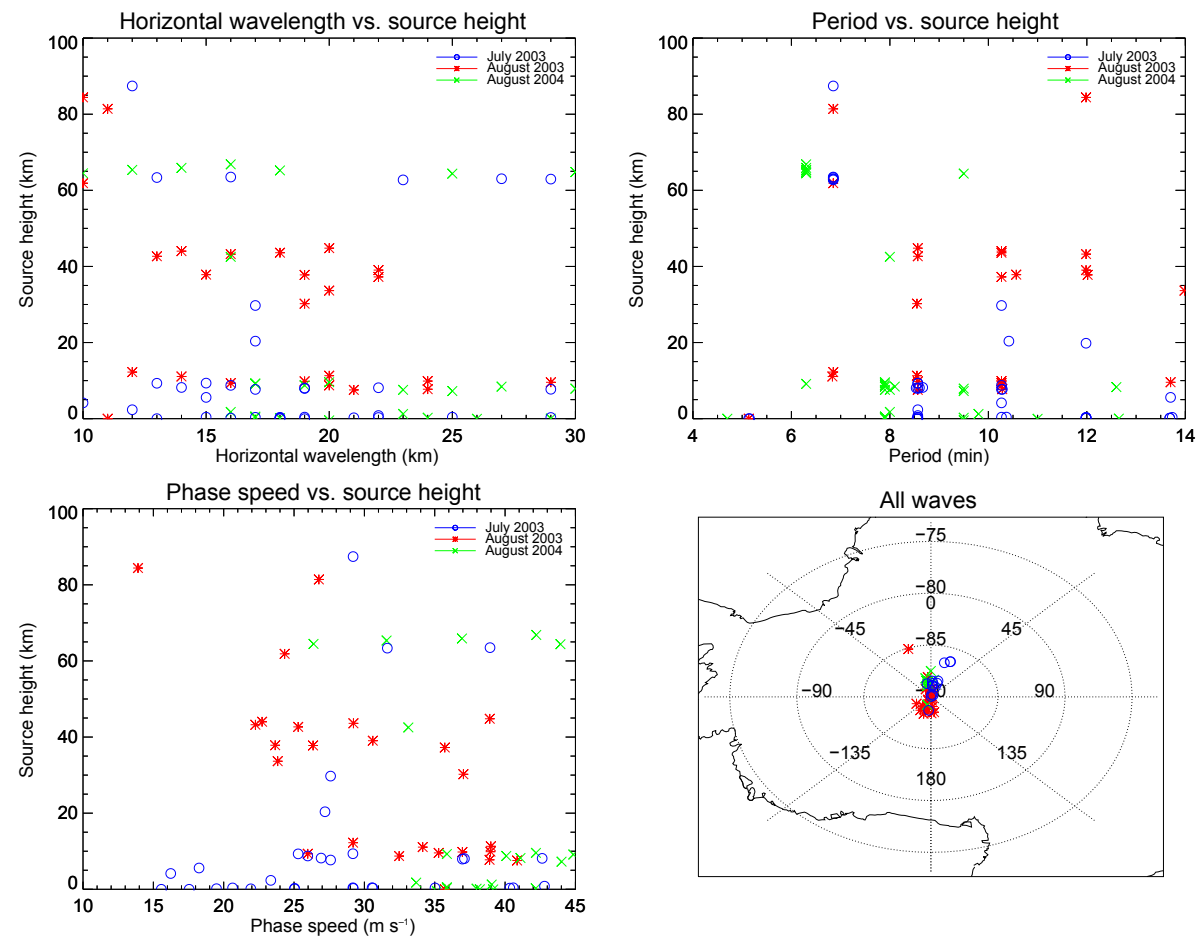

Figure 6. Plots comparing horizontal wavelength (top left), period (top right), and phase speed (bottom left) of the observed waves to the height of their sources as determined by individual GROGRAT runs for the 87 wave events found to be freely propagating waves. The waves are differentiated by month and year, with blue circles representing waves observed during June 2003, red " $x$ " marks denoting waves observed during August 2003, and green "x" marks showing waves observed during August 2004. The bottom right panel shows a plot of the latitude and longitude of the wave sources near the South Pole, from which it is apparent that all but six waves originate within $2.5^{\circ}$ of SPA

tainties in the measurement of wave parameters are around $4.4^{\circ}$ longitude, $2.6^{\circ}$ latitude, and $1.6 \mathrm{~km}$ altitude.

All 94 wave events were ray-traced using GROGRAT, using the background atmospheres constructed from ECMWF reanalyses below $50 \mathrm{~km}$ and NRLMSISE-00 above $50 \mathrm{~km}$. Seven waves were found to be evanescent, indicating they are not propagating gravity waves and are likely to be observations of local convective or dynamical instability processes in the mesopause over SPA. Figure 6 shows plots comparing the source region heights with observed wave parameters for the remaining 87 , freely propagating, waves; 41 of the gravity waves were traced to tropospheric sources, while 16 waves originated above $50 \mathrm{~km}$. As ECMWF does not extend beyond $50 \mathrm{~km}$ altitude, we were unable to analyze the sources of these waves. As shown in Fig. 6, there is no correlation between the height of the wave sources and the spatial and temporal characteristics of the waves. Of the 30 remaining waves, 15 were traced into the tropopause between 9 and $15 \mathrm{~km}$ and the other half into the stratosphere between 15 and $50 \mathrm{~km}$. Based on our results the gravity waves above SPA appear to originate in several discrete layers centered at $65 \mathrm{~km}$, $40 \mathrm{~km}$, the tropopause, and the surface. All but 6 of the waves originated within $2.5^{\circ}$ latitude of SPA, as seen in the bottom right panel of Fig. 6, which shows the distribution of the 87 freely propagating waves around SPA.

\section{Analysis of background source conditions using ECMWF Reanalysis}

In order to identify possible wave-generating regions for our observed waves and modeled wave sources, we examined the background atmospheric conditions around SPA, within the limitations of available data products for the Antarctic lower and middle atmosphere. For this investigation we analyzed $24 \mathrm{~h}$ time-differenced geopotential heights and temperatures obtained from ECMWF Reanalysis from the surface up to $50 \mathrm{~km}$, the upper limit on ECMWF. We mapped $24 \mathrm{~h}$ differenced geopotential heights and temperatures along the wave ray paths as determined by the GROGRAT model runs, as well as in the longitudinal direction opposite from the wave's ray path, such that each slice of data corresponded to a single longitude bin between 0 and $50 \mathrm{~km}$ altitude and $-70^{\circ}$ and $-70^{\circ}$ latitude. By examining $24 \mathrm{~h}$ variations, we are able to see shifts in the structure of the polar vortex towards configurations of high baroclinicity that we would not otherwise be able to as easily infer from the raw geopotential height and temperature maps. Then, by comparing these differenced maps to the wave ray paths, we can determine whether wave sources match regions where baroclinic instabilities or other observable wave source regions are likely to occur. 

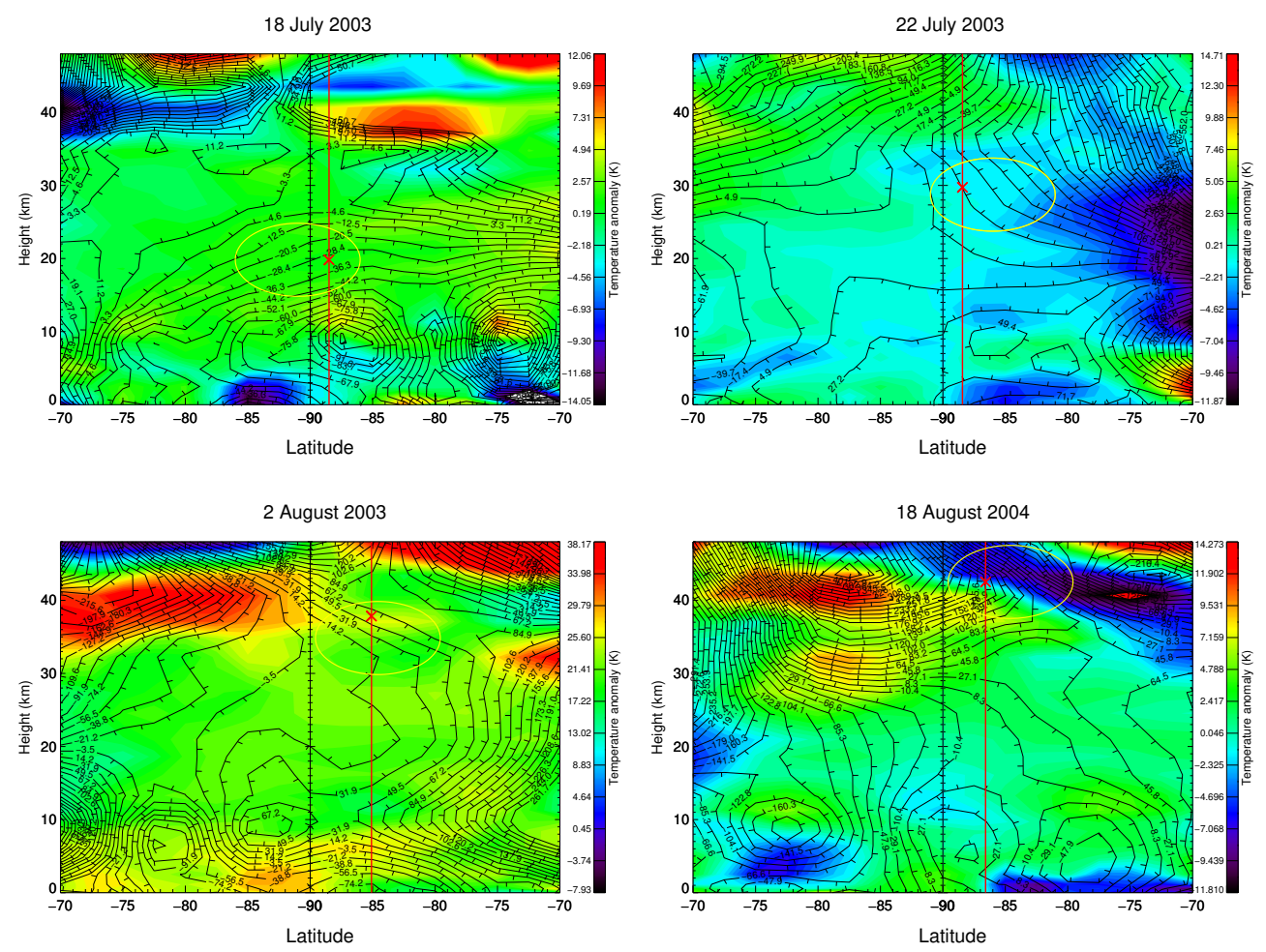

Figure 7. The $24 \mathrm{~h}$ time-differenced contour plots of geopotential height (black contours) and temperatures obtained from ECMWF Reanalysis from 0 to $50 \mathrm{~km}$ along the direction of the ray path for waves observed on 18 July 2003 (top left), 22 July 2003 (top right), 2 August 2003 (bottom left), and 18 August 2004 (bottom right), as determined by our GROGRAT model runs. Ticks on contour lines point to lower geopotential height. Vertical red lines mark the latitude at which the rays terminate, and the corresponding red " $\mathrm{X}$ " denotes the location of the wave source. The yellow oval signifies the region where we infer baroclinic instability.

Figure 7 shows $24 \mathrm{~h}$ time-differenced ECMWF geopotential height and temperature analyses of waves that were found to form in the stratosphere from 18 July 2003, 22 July 2003, 2 August 2003, and 18 August 2004, in regions where the differenced geopotential height maps are heavily slanted latitudinally and vertically, indicating a displacement of the polar vortex that has moved the polar vortex "off-balance" and has likely set up the baroclinic instability that is driving wave excitation. At mid-latitudes a westward tilt is required for a baroclinic wave to draw potential energy from the westerly mean flow (Holton, 1982), but at polar latitudes any displacement from the mean configuration centered over the pole is seen as a generator of gravity waves. Our analysis is further complicated by the lower number of latitude bins near the pole, particularly when one considers that the majority of observed wave sources come from within $2.5^{\circ}$ of SPA. While the direction of tilt can vary latitudinally either towards or away from the pole, this does not appear to affect the formation of the waves, though this may affect the direction of horizontal wave propagation, which would become apparent in a more thorough study over an extended period.

Plots for waves observed on 19 July 2003, 3 and $17 \mathrm{Au}-$ gust 2003, and 9 August 2004 are shown in Fig. 8. These waves form in the tropopause in regions of disturbed geopo- tentials and temperatures. The signature of a planetary wave is present in each case in the vicinity of the wave source, which is the likely cause of the vertical forcing that is generating the waves over SPA. This structure is found in all 15 cases of waves generated in the tropopause.

\section{Discussions and conclusions}

Our observations and model analyses demonstrate that any displacement of the polar vortex, whether locally in the tropopause due to the planetary wave interaction or as a whole in the stratosphere, is sufficient to generate upwardpropagating, and thus upward momentum transporting, gravity waves above the troposphere. However, several questions and concerns still remain. We are limited in terms of the available dataset both due to repeated $>7$-day long gaps for which no $\mathrm{Na}$ airglow data are available as well as the nearconstant presence of auroral contamination in the filter for all UT except the early morning. While there are other days available for the 2003-2005 austral winters, as previously analyzed by Suzuki et al. (2011), these are largely disparate and spread out with larger gaps for which no Na data are avail- 

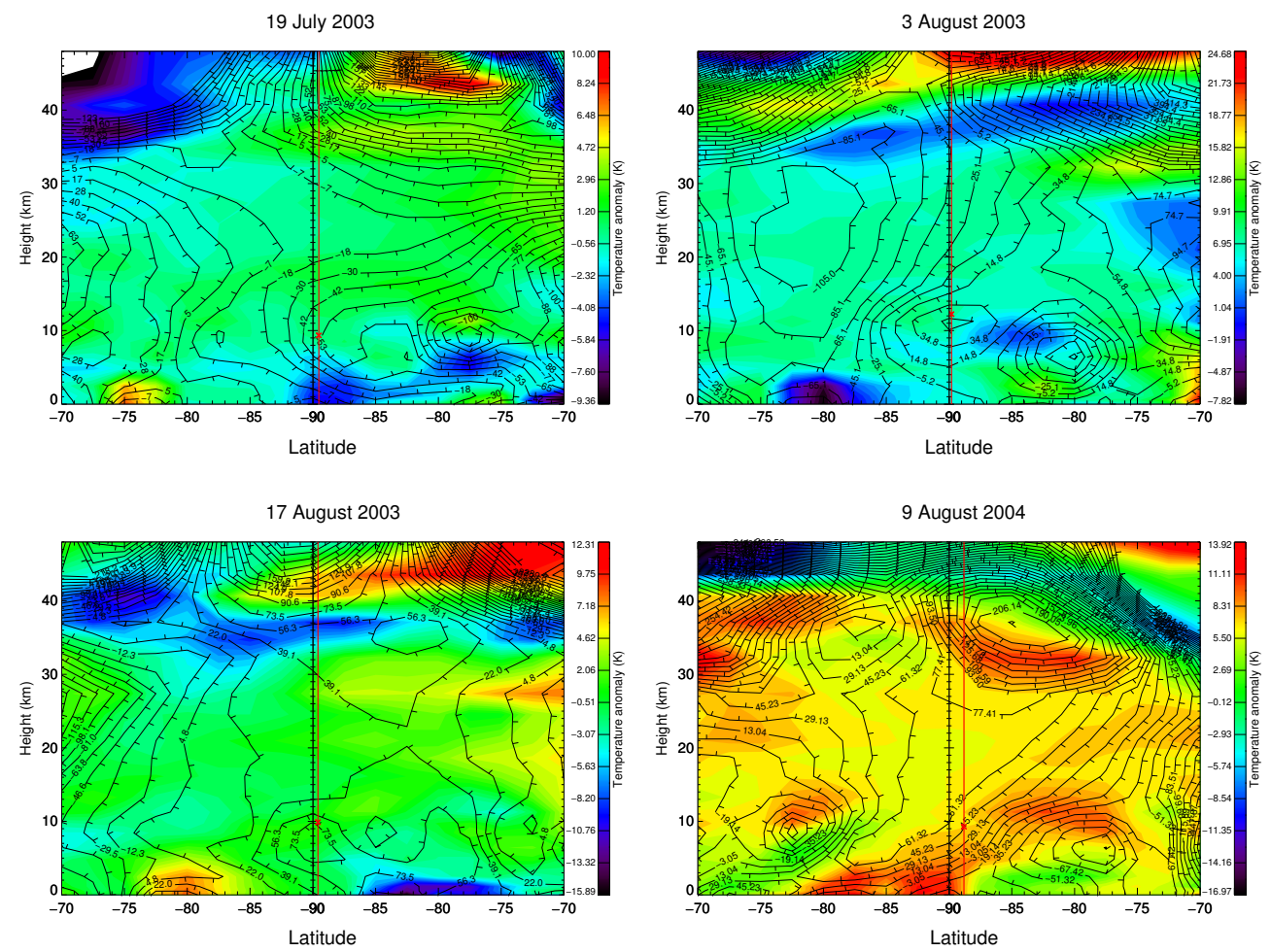

Figure 8. The $24 \mathrm{~h}$ time differenced contour plots of geopotential height (black contours) and temperatures obtained from ECMWF Reanalysis from 0 to $50 \mathrm{~km}$ along the direction of the ray path for waves observed on 19 July 2003 (top left), 3 August 2003 (top right), 17 August 2003 (bottom left), and 9 August 2004 (bottom right), as determined by our GROGRAT model runs. Ticks on contour lines point to lower geopotential height. The vertical red line marks the latitude at which the ray terminates, and the corresponding red " $\mathrm{X}$ " denotes the location of the wave source.
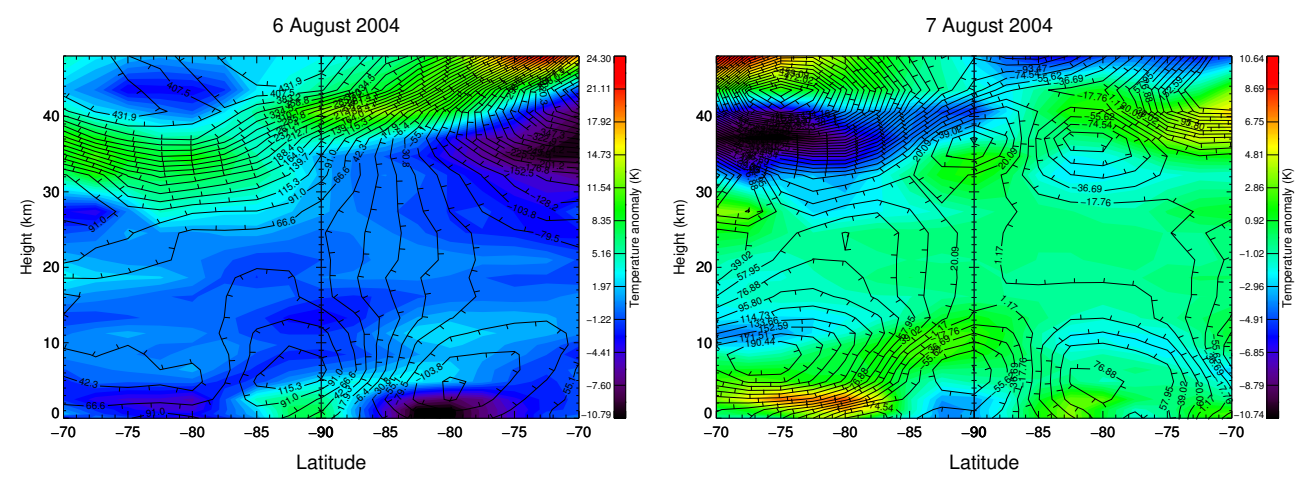

Figure 9. The $24 \mathrm{~h}$ time-differenced contour plots of geopotential height (black contours) and temperatures obtained from ECMWF Reanalysis from 0 to $50 \mathrm{~km}$ along the directions of the ray paths of the 6 August 2004 (left) and 7 August 2004 (right) waves, as determined by GROGRAT. Ticks on contour lines point to lower geopotential height.

able, and thus we have ignored these for now, focusing on periods of continuous observation over $\sim 7$-day intervals.

Due to the rapidly changing background atmospheric conditions responsible for gravity wave excitation, and our reliance on NRLMSISE-00 and HWM-93 climatologies above $50 \mathrm{~km}$, we are able to analyze the results of the ray-tracing runs with ray paths terminating in the mesosphere to only a limited extent. Two examples of this are runs for 6 and $7 \mathrm{Au}-$ gust 2004, where the wave rays originated at $65 \mathrm{~km}$. Differenced geopotential and temperature plots for these two cases are shown in Fig. 9. As the polar vortex extends upward into the MLT, the apparent disturbance of the polar vortex below $50 \mathrm{~km}$ seen in both figures should similarly extend upward, and is likely to be the source of the waves we observed over the SPA site. However, without the availability of a model that can account for synoptic-scale variation for the polar 
mesosphere for this time period, we are unable to further our analysis. This is unfortunate, as waves in this region account for 16 of the 87 waves found by our model to be real, propagating waves, and this is roughly equal in number to the waves originating from the stratosphere or tropopause.

Another consideration is our current reliance on model winds for the characterization of gravity wave intrinsic frequencies and vertical wave numbers, both necessary components as inputs into GROGRAT. Any divergence of the real background winds from the model represents a source of error for our model runs, though with winds typically being low near the pole during winter this is not expected to be a large error source. While a real vertical wind profile over SPA would be ideal, the inclusion of available meteor radar winds at $95 \mathrm{~km}$ could resolve this problem; however, at the present time we have elected not to include these data, as we are unable to adequately constrain the winds with a single point measurement at $95 \mathrm{~km}$.

In this paper, we have shown through the combination of observation and numerical modeling that the polar tropopause and stratosphere are frequent sources of upwardpropagating gravity waves. While there are inherent limitations to our analysis both in terms of available image and atmospheric data and in refining our modeling efforts with additional, existing data, we have presented a compelling case for a previously unidentified source of small-scale gravity waves in the polar MLT.

Previous analyses of the Arctic polar vortex by Bhattacharya and Gerrard (2010) have looked at the response of the polar vortex during quiet conditions to drivers in the MLT as a form of downward control by thermospheric winds. These winds are known to, in turn, respond to variations in gravity wave input into the region. With both upward and downward energy transport affecting dynamics throughout the lower and middle atmosphere, we are left with an extensive coupled system with built-in feedback mechanisms. The excitation of gravity waves in the tropopause and stratosphere by the establishment of baroclinic instabilities through displacement of the polar vortex is an important component in the system in need of further study.

\section{Data availability}

All data resources used in this study are mentioned in the acknowledgments below.

Acknowledgement. This study was supported by the National Science Foundation with grants PLR-1247975 and 942 PLR1443507 to the New Jersey Institute of Technology. Access to SPA imager data can be obtained from http://www.southpole-aurora.org. ECMWF TOGA analyses can be obtained from the National Center for Atmospheric Research (NCAR) Research Data Archives (RDA) from http://rda.ucar.edu/datasets/ds111.2/. NRLMSISE-00 can be obtained from http://ccmc.gsfc.nasa.gov/modelweb/models/ nrlmsise00.php, and the horizontal wind model is available at $\mathrm{ftp}$ : //hanna.ccmc.gsfc.nasa.gov/pub/modelweb/atmospheric/hwm93/, both from the NASA Community Coordinated Modeling Center (CCMC).

Edited by: M. Dameris

Reviewed by: three anonymous referees

\section{References}

Bhattacharya, Y. and Gerrard, A. J.: Correlations of mesospheric winds with subtle motion of the Arctic polar vortex, Atmos. Chem. Phys., 10, 431-436, doi:10.5194/acp-10-431-2010, 2010.

Brown, L., Gerrard, A., Meriwether, J., and Makela, J.: All-sky imaging observations of mesospheric fronts in OI $557.7 \mathrm{~nm}$ and broadband $\mathrm{OH}$ airglow emissions: Analysis of frontal structure, atmospheric background conditions, and potential sourcing mechanisms, J. Geophys. Res., 109, D19104, doi:10.1029/2003JD004223, 2004.

Chen, C., Chu, X. McDonald, A. J., Vadas, S. L., Yu, Z., Fong, W., and Lu, X.: Inertia-gravity waves in Antarctica: A case study using simultaneous lidar and radar measurements at McMurdo/Scott Base $\left(77.8^{\circ} \mathrm{S}, 166.7^{\circ} \mathrm{E}\right)$, J. Geophys. Res., 118, 2794-2808, doi:10.1002/jgrd.50318, 2013.

Chu, X., Yu, Z., Gardner, C. S., Chen, C., and Fong, W.: Lidar observations of neutral $\mathrm{Fe}$ layers and fast gravity waves in the thermosphere $(110-155 \mathrm{~km})$ at McMurdo $\left(77.8^{\circ} \mathrm{S}, 166.7^{\circ} \mathrm{E}\right)$, Antarctica, Geophys. Res. Lett., 38, L23807, doi:10.1029/2011GL050016, 2011.

Duck, T. J., Whiteway, J. A., and Carswell, A. I.: Lidar observations of gravity wave activity and Arctic stratospheric vortex core warming, Geophys. Res. Lett., 25, 2813-2816, 1998.

Dunkerton, T. J. and Butchart, N.: Propagation and selective transmission of internal gravity waves in a sudden warming, J. Atmos. Sci., 41, 1443-1460, 1984.

Eckermann, S. D. and Marks, C. J.: GROGRAT: A new model of the global propagation and dissipation of atmospheric gravity waves, Adv. Space Res., 20, 1253-1256, 1997.

Ejiri, M., Aso, T., Okada, M., Tsutsumi, M., Taguchi, M., Sato, N., and Okano, S.: Japanese research project on Arctic and Antarctic observations of the middle atmosphere, Adv. Space Res., 24, 1689-1692, 1999.

European Centre for Medium-Range Weather Forecasts: ECMWF TOGA 2.5 degree Global Surface and Upper Air Analyses, available at: http://rda.ucar.edu/datasets/ds111.2/ (last access: 30 April 2015), 1990.

Fairlie, T., Fisher, M., and O'Neill, A.: The development of narrow baroclinic zones and other small-scale structure in the stratosphere during simulated major warmings, Q. J. Roy. Meteor. Soc., 116, 287-315, 1990.

Fritts, D. C. and Alexander, M. J.: Gravity wave dynamics and effects in the middle atmosphere, Rev. Geophys., 41, 1003, doi:10.1029/2001RG000106, 2003.

Garcia, F., Taylor, M. J., and Kelley, M.: Two-dimensional spectral analysis of mesospheric airglow image data, Appl. Optics, 36, 7374-7385, 1997.

Gerrard, A. J., Kane, T. J., Thayer, J. P., Duck, T. J., Whiteway, J. A., and Fiedler, J.: Synoptic scale study of the arctic polar vortex's 
influence on the middle atmosphere, 1, observations, J. Geophys. Res., 107, ACL 1-1-ACL 1-15, doi:10.1029/2001JD000681, 2002.

Gerrard, A. J., Kane, T. J., Eckermann, S. D., and Thayer, J. P.: Gravity waves and mesospheric clouds in the summer middle atmosphere: A comparison of lidar measurements and ray modeling of gravity waves over Sondrestrom, Greenland, J. Geophys. Res., 109, D10103, doi:10.1029/2002JD002783, 2004.

Gerrard, A. J., Bhattacharya, Y., and Thayer, J. P.: Observations of in-situ generated gravity waves during a stratospheric temperature enhancement (STE) event, Atmos. Chem. Phys., 11, 1191311917, doi:10.5194/acp-11-11913-2011, 2011.

Guest, F. M., Reeder, M. J., Marks, C. J., and Karoly, D. J.: Inertiagravity waves observed in the lower stratosphere over Macquarie Island, J. Atmos. Sci., 57, 737-752, 2000.

Hedin, A. E., Fleming, E., Manson, A., Schmidlin, F., Avery, S., Clark, R., Franke, S., Fraser, G., Tsuda, T., Vial, F., and Vincent, R. A.: Empirical wind model for the upper, middle and lower atmosphere, J. Atmos. Terr. Phys., 58, 1421-1447, 1996.

Holton, J. R.: The role of gravity wave induced drag and diffusion in the momentum budget of the mesosphere, J. Atmos. Sci., 39, 791-799, 1982.

Kaifler, B., Lübken, F.-J., Höffner, J., Morris, R. J., and Viehl, T. P.: Lidar observations of gravity wave activity in the middle atmosphere over Davis $\left(69^{\circ} \mathrm{S}, 78^{\circ} \mathrm{E}\right)$, Antarctica, J. Geophys. Res., 120, 4506-4521, 2015.

Lane, T. P., Doyle, J. D., Plougonven, R., Shapiro, M. A., and Sharman, R. D.: Observations and numerical simulations of inertiagravity waves and shearing instabilities in the vicinity of a jet stream, J. Atmos. Sci., 61, 2692-2706, 2004.

Li, Z., Naqvi, S., Gerrard, A. J., Chau, J. L., and Bhattacharya, Y.: Initial MST radar observations of upper troposphericlower stratospheric duct-like structures over Jicamarca, Peru, Atmos. Chem. Phys., 12, 11085-11093, doi:10.5194/acp-1211085-2012, 2012.

Lighthill, J.: Waves in Fluids, Cambridge Univ. Press, New York, 504 pp., 1978.

Lin, Y. and Zhang, F.: Tracking gravity waves in baroclinic jet-front systems, J. Atmos. Sci., 65, 2402-2415, 2008.

Marks, C. J. and Eckermann, S. D.: A three-dimensional nonhydrostatic ray-tracing model for gravity waves: Formulation and preliminary results for the middle atmosphere, J. Atmos. Sci., 52, 1959-1984, 1995.

Meriwether, J. W. and Gerrard, A. J.: Mesosphere inversion layers and stratosphere temperature enhancements, Rev. Geophys., 42, RG3003, doi:10.1029/2003RG000133, 2004.

Moffat-Griffin, T., Hibbins, R. E., Jarvis, M. J., and Colwell, S. R.: Seasonal variations of gravity wave activity in the lower stratosphere over an Antarctic Peninsula station, J. Geophys. Res., 116, D14111, doi:10.1029/2010JD015349, 2011.

Nielsen, K., Taylor, M. J., Hibbins, R., Jarvis, M., and Russell, J.: On the nature of short-period mesospheric gravity wave propagation over Halley, Antarctica, J. Geophys. Res., 117, D05124, doi:10.1029/2011JD016261, 2012.
O’Sullivan, D. and Dunkerton, T. J.: Generation of inertia-gravity waves in a simulated life cycle of baroclinic instability, J. Atmos. Sci., 52, 3695-3716, 1995.

Oyama, S. and Watkins, B.: Generation of atmospheric gravity waves in the polar thermosphere in response to auroral activity, Space Sci. Rev., 168, 463-473, 2012.

Picone, J., Hedin, A., Drob, D. P., and Aikin, A.: NRLMSISE00 empirical model of the atmosphere: Statistical comparisons and scientific issues, J. Geophys. Res., 107, 1468, doi:10.1029/2002JA009430, 2002.

Plougonven, R. and Snyder, C.: Inertia-gravity waves spontaneously generated by jets and fronts. Part I: Different baroclinic life cycles, J. Atmos. Sci., 64, 2502-2520, 2007.

Plougonven, R., Teitelbaum, H., and Zeitlin, V.: Inertia gravity wave generation by the tropospheric midlatitude jet as given by the Fronts and Atlantic Storm-Track Experiment radio soundings, J Geophys. Res., 108, 4686, doi:10.1029/2003JD003535, 2003.

Sato, K. and Yoshiki, M.: Gravity wave generation around the polar vortex in the stratosphere revealed by 3-hourly radiosonde observations at Syowa Station, J. Atmos. Sci., 65, 3719-3735, 2008.

Suzuki, S., Tsutsumi, M., Palo, S., Ebihara, Y., Taguchi, M., and Ejiri, M.: Short-period gravity waves and ripples in the South Pole mesosphere, J. Geophys. Res., 116, D19109, doi:10.1029/2011JD015882, 2011.

Tanaka, H. and Tokinaga, H.: Baroclinic instability in high latitudes induced by polar vortex: A connection to the Arctic Oscillation, J. Atmos. Sci., 59, 69-82, 2002.

Vadas, S. L., Taylor, M. J., Pautet, P.-D., Stamus, P. A., Fritts, D. C., Liu, H.-L., São Sabbas, F. T., Rampinelli, V. T., Batista, P., and Takahashi, H.: Convection: the likely source of the medium-scale gravity waves observed in the $\mathrm{OH}$ airglow layer near Brasilia, Brazil, during the SpreadFEx campaign, Ann. Geophys., 27, 231-259, doi:10.5194/angeo-27-231-2009, 2009.

Wang, L. and Alexander, M. J.: Gravity wave activity during stratospheric sudden warmings in the 2007-2008 Northern Hemisphere winter, J. Geophys. Res., 114, D18108, doi:10.1029/2009JD011867, 2009.

Whiteway, J. A. and Duck, T. J.: Enhanced Arctic stratospheric gravity wave activity above a tropospheric jet, Geophys. Res. Lett., 26, 2453-2456, 1999.

Yamashita, C., Liu, H.-L., and Chu, X.: Responses of mesosphere and lower thermosphere temperatures to gravity wave forcing during stratospheric sudden warming, Geophys. Res. Lett., 37, L09803, doi:10.1029/2009GL042351, 2010.

Yamashita, C., England, S. L., Immel, T. J., and Chang, L. C.: Gravity wave variations during elevated stratopause events using SABER observations, J. Geophys. Res., 118, 5287-5303, 2013. 